\title{
Acoustoelectric interaction in degenerately doped piezoelectric semiconductors
}

\author{
Mosekilde, Erik
}

Published in:

Journal of Applied Physics

Link to article, DOI:

$10.1063 / 1.1661053$

Publication date:

1972

Document Version

Publisher's PDF, also known as Version of record

Link back to DTU Orbit

Citation (APA):

Mosekilde, E. (1972). Acoustoelectric interaction in degenerately doped piezoelectric semiconductors. Journal of Applied Physics, 43(12), 4957-4963. https://doi.org/10.1063/1.1661053

\section{General rights}

Copyright and moral rights for the publications made accessible in the public portal are retained by the authors and/or other copyright owners and it is a condition of accessing publications that users recognise and abide by the legal requirements associated with these rights.

- Users may download and print one copy of any publication from the public portal for the purpose of private study or research.

- You may not further distribute the material or use it for any profit-making activity or commercial gain

- You may freely distribute the URL identifying the publication in the public portal

If you believe that this document breaches copyright please contact us providing details, and we will remove access to the work immediately and investigate your claim 


\title{
Acoustoelectric interaction in degenerately doped piezoelectric semiconductors
}

\author{
E. Mosekilde \\ Physics Laboratory III, The Technical University of Denmark, Lyngby, Denmark \\ (Received 13 September 1971) \\ The acoustoelectric gain constant is calculated quantum mechanically for arbitrary degeneracy of the \\ electron gas. The requirements of energy and momentum conservation in individual electron-phonon \\ interactions result in a rather sharp reduction of the gain factor when the acoustic wave number exceeds \\ the characteristic linear extension of the electron distribution in reciprocal space. A simple two-band \\ model is developed to describe effects of impurity banding upon the acoustoelectric interaction. \\ According to this model, impurity banding enhances the acoustoelectric coupling and widens the active \\ acoustic spectrum. These effects are associated with the increased density of states near the \\ conduction-band edge and with the presence of electrons of higher momentum effective mass than the \\ conduction-band electrons. At low temperatures it becomes possible for the linear gain factor as a \\ function of acoustic frequency to have two maxima.
}

\section{INTRODUCTION}

In two recent papers, ${ }^{1,2}$ the use of $x$-ray diffraction to obtain the spectral distribution of piezoelectrically amplified sound flux at very high acoustic frequencies was reported. The $x$-ray scattering technique for the first time permits a detailed investigation of the quantum regime of acoustoelectric interaction in which the acoustic wavelength becomes comparable to a characteristic electron deBroglie wavelength.

The first experiments ${ }^{1}$ were performed at $77 \mathrm{~K}$ using an epitaxial single crystal of GaAs with a free-carrier density of $4 \times 10^{16} \mathrm{~cm}^{-3}$. At $77 \mathrm{~K}$, the effective density of states for the conduction band of GaAs is $N_{c}=5.4$ $\times 10^{16} \mathrm{~cm}^{-3}$, and the electron gas of the sample was the refore partly degenerate. In the following experiments, ${ }^{2}$ the doping was increased to $1.35 \times 10^{17} \mathrm{~cm}^{-3}$, and the temperature reduced to $20 \mathrm{~K}$ so as to approach a fully degenerate electron gas. A Kohn-type anomaly ${ }^{3,4}$ was then observed in the form of a dramatic decrease in acoustic gain for acoustic wave numbers $q$ larger than twice the radius of the electron Fermi sphere $k_{F}$. An additional anomaly consisting of the apparent presence of two maxima in the spectral distribution of the amplified acoustic flux was attributed to parametric interactions among the amplified lattice modes.

For nondegenerate electron statistics, Nakamura, ${ }^{5}$ Paranjape, ${ }^{6}$ and Conwell ${ }^{7}$ have discussed the acoustoelectric interaction quantum mechanically in terms of stimulated phonon emission. In the present paper, these calculations are extended to permit arbitrary degeneracy of the electron gas. An alternative procedure ${ }^{8,2}$ is to evaluate the acoustic attenuation constant from the dielectric response function as obtained from a quantum-mechanical density matrix calculation. Independent of the degree of degeneracy, the quantum treatment predicts a fairly sharp cutoff of the acoustoelectric interaction when the acoustic wave number exceeds the characteristic linear extension of the electron distribution in reciprocal space. For a fully degenerate electron gas, the cutoff is practically abrupt, reflecting the sharpness of the Fermi surface.

In Sec. II, we discuss the acoustoelectric interaction for degenerately doped piezoelectric semiconductors in a rigid-band model which assumes the intrinsic band structure of the material to remain unaffected by the presence of impurities. All free electrons are thus ascribed to the conduction band, for which an effective mass approximation is employed.

At high doping levels, the overlap of bound electron wave functions associated with neighboring donor sites may lead to the formation of an impurity band ${ }^{9}$ in which charge transport can take place. To describe the effects of impurity banding upon the acoustoelectric interaction, we consider in Sec. III a simple two-band model for the electronic states. The intrinsic conduction band of the material and the impurity band are here both described in the effective mass approximation.

According to our model, the presence of an impurity band leads to an enhancement of the acoustoelectric coupling and to a broadening of the active frequency range. At low temperatures where the separation between conduction band and impurity band is large compared to thermal energy, it becomes possible for the gain factor as a function of frequency to have two maxima. Impurity banding therefore offers an alternative explanation to mode conversion of the broadening of the acoustic spectrum as well as of the double peak reported in Refs. 1 and 2, respectively.

\section{ACOUSTOELECTRIC INTERACTION WITH ARBITRARY DEGENERACY}

In this section, we discuss the acoustoelectric interaction for a piezoelectric semiconductor with arbitrarily degenerate electron gas. It is assumed that the ef fective mass approximation applies. The electronphonon coupling is described in terms of single phonon emission and phonon absorption processes which conserve energy and momentum. The net rate of phonon emission is obtained by integrating the result for individual electron-phonon interactions over the complete electron distribution in momentum space.

The external drift field, assumed to be applied along the direction of acoustic propagation, gives rise to a displacement of the electron distribution in momentum space by $\hbar k_{d}=m^{*} v_{d}$. Here $m^{*}$ is the effective mass of the free electrons, $v_{d}$ the drift velocity, and $\hbar$ Dirac's constant. At low drift fields, the electron distribution is such that the lattice mode will be damped by the interaction with the free carriers. If the displacement of the electron distribution in momentum space exceeds $m^{*} v_{s}$, however, the electron population becomes in- 
verted relative to acoustic phonons, and net phonon emission will result. ${ }^{10} v_{s}$ is the velocity of sound.

We consider a single highly excited lattice mode $\exp [i(q x-\omega t)]$ propagating along the $x$ axis of our coordinate system and interacting with a drifted Fermi distribution of conduction electrons. $q$ is the acoustic wave number and $\omega$ the angular acoustic frequency. Conservation of energy and momentum ${ }^{11}$ requires that the $x$ component of the initial and final electron momentum in a phonon emission (upper sign) or absorption (lower sign) process be given by ${ }^{12}$

$$
\hbar k_{x i}=m^{*} v_{s} \pm \frac{1}{2} \hbar q
$$

and

$$
\hbar k_{x f}=m^{*} v_{s} \mp \frac{1}{2} \hbar q,
$$

respectively.

Using first-order perturbation theory, the net rate of phonon emission is calculated to be ${ }^{12}$

$P=(2 \pi / \hbar) \sum_{\mathbf{k}}\left|H_{\mathbf{k}+\mathbf{q}, \mathbf{k}}\right|^{2}[f(\mathbf{k}+\mathbf{q})-f(\mathbf{k})] \delta\left(E_{\mathbf{k}+\mathbf{q}}-E_{\mathbf{k}}-\hbar \omega\right)$,

where the summation extends over allowed electron wave vectors $\mathbf{k}$. $f(\mathbf{k})$ is the electron distribution function giving the probability that a state $\mathrm{k}$ of energy $E_{\mathrm{k}}$ be occupied. The squared modulus of the matrix element for electron-phonon interaction is given by ${ }^{13}$

$$
\left|H_{\mathbf{k}+\mathbf{q}, \mathbf{k}}\right|^{2} \equiv|H|^{2}=N_{q} \hbar q C^{2} / 2 v_{s} \rho V,
$$

where $N_{q}$ is the phonon occupation number, $C$ is the screened electron-phonon coupling constant, $\rho$ the mass density of the material, and $V$ the sample volume.

By means of the energy conserving $\delta$ function, the summation over allowed states in reciprocal space can be transformed into an integration over the interaction plane $S$ defined by ${ }^{10}$

$$
k_{x}=\frac{1}{2}\left(k_{x i}+k_{x f}\right)=m^{*} v_{s} / \hbar
$$

The net rate of phonon emission will then be given by

$$
P=\left(m^{*} V|H|^{2} / 2 \pi^{2} \hbar^{3} q\right) \int_{S}\left[f\left(\mathbf{k}+\frac{1}{2} \mathrm{q}\right)-f\left(\mathrm{k}-\frac{1}{2} \mathrm{q}\right)\right] d^{2} k \text {. }
$$

The electron distribution function with a drift field applied along the $x$ axis is taken to be the displaced Fermi-Dirac distribution

$$
f(\mathrm{k})=\left[\exp \left(\frac{\hbar^{2}}{2 m^{*} k_{\mathrm{B}} T}\left[\left(k_{x}-k_{d}\right)^{2}+k_{y}^{2}+k_{z}^{2}\right] \mid-\frac{E_{F}}{k_{B} T}\right)+1\right]^{-1},
$$

where $E_{F}$ is the Fermi energy as measured from the conduction-band edge and $k_{B} T$ is the thermal energy. The displacement of the electron distribution in reciprocal space is $k_{a}=m^{*} v_{a} / \hbar$, where $v_{a}$ is the electron drift velocity. The assumption of a drifted thermal equilibrium distribution is usually justified in heavily doped semiconductors due to relatively strong electron-electron scattering. The neglect of the energy dependence of the carrier momentum relaxation time involved in such an assumption is further justified by the degeneracy of the electron gas. For nondegenerate semiconductors, on the other hand, this neglect can lead to errors as large as $100 \%$ in the acoustoelectric absorption constant, as has been shown in Jacoboni and Prohofsky. ${ }^{14}$
Assuming the electron drift velocity $v_{d}$ and the velocity of sound $v_{s}$ both to be much smaller than the thermal velocity of the free electrons, $v_{T}=\left(2 k_{B} T / m^{*}\right)^{1 / 2}$, an expansion to first order in the quantity $\left(v_{s}-v_{d}\right) / v_{T}$ gives

$$
f\left(\mathbf{k}+\frac{1}{2} \mathbf{q}\right)-f\left(\mathbf{k}-\frac{1}{2} \mathbf{q}\right)=2 A e^{u}\left(e^{u}+1\right)^{-2},
$$

with

$$
\begin{aligned}
& u=\hbar^{2}\left(\frac{1}{4} q^{2}+k_{y}^{2}+k_{z}^{2}\right) / 2 m * k_{B} T-E_{F} / k_{B} T, \\
& A=\hbar q\left(v_{d}-v_{s}\right) / 2 k_{B} T .
\end{aligned}
$$

The integration over the interaction plane can now easily be performed. We obtain

$$
P=\left(m^{*}\right)^{2} V|H|^{2}\left(v_{d}-v_{s}\right) f_{0}(a) / \pi \hbar^{4},
$$

with

$$
\begin{aligned}
& a=\hbar^{2} q^{2} / 8 m * k_{B} T, \\
& f_{0}(a)=[\exp (a-\eta)+1]^{-1} .
\end{aligned}
$$

$\eta=E_{F} / k_{B} T$ is the normalized Fermi energy. $f_{0}(a)$ is the usual Fermi factor giving the probability that an electron state with wave number $\frac{1}{2} q$ is occupied. This factor arises because an electron must have a minimum wave number of (approximately) $\frac{1}{2} q$ to emit a phonon of wave number $q$ in an energy and momentum conserving process. At high phonon wave numbers, only a fraction of the free electrons have sufficient momentum for phonon emission, and the net rate of emission is correspondingly reduced.

In the Thomas-Fermi approach, ${ }^{12}$ the screened piezoelectric coupling constant $C$ can be expressed in terms of the unscreened coupling constant $C_{p}=e p / \epsilon q$ by

$$
C=C_{p} q^{2} /\left(q^{2}+q_{0}^{2}\right) .
$$

$e$ is here the elementary charge, $p$ the piezoelectric constant, and $\epsilon$ the static dielectric constant. $q_{0}$ is the screening wave number determined by

$$
q_{0}^{2}=\frac{e^{2}}{\epsilon} \frac{\partial n_{c}}{\partial E_{F}}
$$

where $n_{c}$ is the density of free electrons. In the effective mass approximation we have $e^{15}$

$$
\begin{aligned}
& n_{c}=N_{c} F_{1 / 2}(\eta), \\
& \frac{\partial n_{c}}{\partial E_{F}}=\frac{N_{c} F_{-1 / 2}(\eta)}{k_{B} T},
\end{aligned}
$$

where $N_{c}=2\left(m^{*} k_{B} T / 2 \pi \hbar^{2}\right)^{3 / 2}$ is the effective density of states for the conduction band. $F_{1 / 2}(\eta)$ and $F_{-1 / 2}(\eta)$ belong to a class of Fermi integrals defined by ${ }^{15}$

$$
F_{j}(\eta)=\frac{1}{\Gamma(j+1)} \int_{0}^{\infty} \frac{x^{j} d x}{\exp (x-\eta)+1}
$$

where $\Gamma(j+1)$ is the usual gamma function. The squared screening wave number for a semidegenerate electron gas is then calculated to be ${ }^{16}$

$$
q_{0}^{2}=\left(N_{c} e^{2} / \epsilon k_{B} T\right) F_{-1 / 2}(\eta)=q_{D}^{2} F_{-1 / 2}(\eta) / F_{1 / 2}(\eta) .
$$

$q_{D}=\left(n_{c} e^{2} / \epsilon k_{B} T\right)^{1 / 2}$ is here the Debye screening wave number.

Quantum mechanically calculated, the screening wave number itself is a function of acoustic wave number. 4,8 Expression (19) only gives the low-frequency limit for 


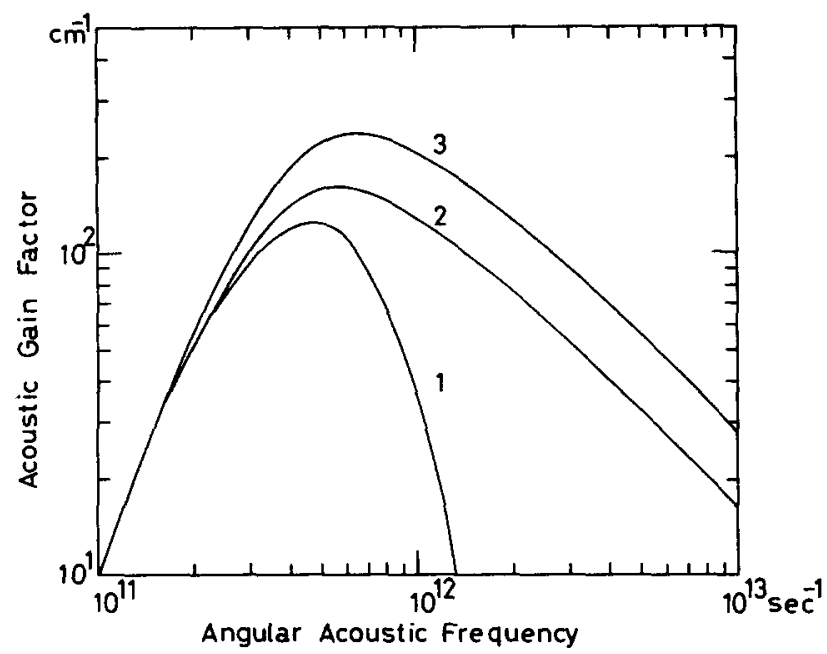

FIG. 1. Acoustic gain factor as a function of angular frequency for fast transverse waves propagating along the [110] direction of GaAs with a carrier density of $5.4 \times 10^{16} \mathrm{~cm}^{-3}$ at $77 \mathrm{~K}$. The curves were calculated quantum mechanically including the partial degeneracy of the electron gas (curve 1), semiclassically including degeneracy (curve 2), and semiclassically neglecting degeneracy (curve 3). The quantum theory predicts a cutoff of the acoustoelectric interaction when the acoustic wave number exceeds the linear extension of the electron distribution in reciprocal space.

$q_{0}$. As the acoustic wave number exceeds the characteristic linear extension of the electron distribution in reciprocal space, the screening wave number starts to decrease and approaches zero at very high frequencies. This occurs because, as the acoustic wave number is increased, fewer and fewer electrons can be excited by the acoustic wave. The variation of the screening wave number with $q$, however, is a second-order effect compared with the direct reduction of the net rate of phonon emission at high acoustic frequencies through the factor $f_{0}(a)$. In the present discussion we shall therefore take the screening wave number to be independent of acoustic wave number.

Inserting now the matrix element for electron-phonon interaction into expression (11) for the net rate of phonon emission, we obtain for the acoustoelectric gain factor

$$
\beta=\frac{P}{v_{s} N_{q}}=\sqrt{\pi} \frac{K^{2} \omega}{v_{T}} \frac{q^{2} q_{c}^{2}}{\left(q^{2}+q_{0}^{2}\right)^{2}} f_{0}(a)\left(\frac{v_{d}}{v_{s}}-1\right),
$$

with

$$
q_{c}^{2}=e^{2} N_{c} / \epsilon k_{B} T .
$$

$K$ is here the electromechanical coupling constant, $K^{2}$ $=p^{2} / \epsilon c, c$ being the elastic constant.

In the limit of nondegenerate statistics, the expression for the gain factor reduces to

$$
\beta=\sqrt{\pi} \frac{K^{2} \omega}{v_{T}} \frac{q^{2} q_{D}^{2}}{\left(q^{2}+q_{D}^{2}\right)^{2}} e^{-a}\left(\frac{v_{d}}{v_{s}}-1\right),
$$

an expression which has previously been obtained by Nakamura. ${ }^{5}$ For a fully degenerate electron gas, the gain factor becomes

$$
\beta=\frac{\pi}{2} \frac{K^{2} \omega}{v_{F}} \frac{q^{2} q_{T}^{2}}{\left(q^{2}+q_{T}^{2}\right)^{2}} \theta(\eta-a)\left(\frac{v_{d}}{v_{s}}-1\right),
$$

where $q_{T}=\left(3 n_{c} e^{2} / 2 \epsilon E_{F}\right)^{1 / 2}$ is the Thomas-Fermi screen ing wave number, and $v_{F}=\left(2 E_{F} / m^{*}\right)^{1 / 2}$ is the Fermi velocity of the free electrons. Omitting the step function

$$
\begin{aligned}
\theta(\eta-a) & =1 \text { for } \eta>a, \\
& =0 \text { for } \eta<a,
\end{aligned}
$$

a similar expression for the gain factor with fully degenerate statistics was previously given by McFee. ${ }^{12}$ The function $\theta(\eta-a)$ reflects the fact that for a totally degenerate electron gas, acoustic modes with wave numbers greater than $2 k_{F}$ do not interact with the conduction electrons in energy and momentum conserving processes. ${ }^{3,8} k_{F}=\left(2 m^{*} E_{F} / \hbar^{2}\right)^{1 / 2}$ is the radius of the Fermi sphere.

The parameter $a=\hbar^{2} q^{2} / 8 m^{*} k_{B} T$ can be expressed in terms of the ratio between the thermal electron deBroglie wavelength $\lambda_{e}=2 \pi \hbar /\left(2 m^{*} k_{B} T\right)^{1 / 2}$ and the acoustic wavelength $\lambda_{\mathrm{ac}}=2 \pi / q$, i.e., $a=\left(\lambda_{e} / 2 \lambda_{\mathrm{ac}}\right)^{2}$. As long as the acoustic wavelength is much longer than the electron deBroglie wavelength, $a$ can be neglected, and expres sion (20) for the acoustoelectric gain factor reduces to the expression that one would calculate for a semidegenerate electron gas using the microscopic Boltzmannequation method. ${ }^{17} \mathrm{~A}$ semiclassical microscopic calculation of the acoustoelectric gain factor for arbitrary degeneracy seems prohibitively complicated, however. When $\lambda_{\mathrm{ac}}$ becomes comparable to $\lambda_{e}$, the electron recoil in a single phonon scattering process becomes significant. A quantum-mechanical correction to the microscopic theory then arises which leads to the gain factor becoming proportional to the probability of occupancy for an electron state of kinetic energy $a k_{B} T$, rather than the probability of occupancy at the conduction-band edge.

To illustrate the effects of the quantum correction and of the partial degeneracy of the electron gas, we have plotted in Fig. 1 (curve 1) the acoustoelectric gain factor $\beta$ as a function of angular acoustic frequency $\omega$ for a GaAs sample with a free-carrier density of $n_{c}=5.4$ $\times 10^{16} \mathrm{~cm}^{-3}\left(=N_{c}\right)$ at $77 \mathrm{~K}$. For the effective electron mass and the squared electromechanical coupling constant, we have assumed $m^{*}=0.067 m_{0}$ and $K^{2}=3.7$ $\times 10^{-3}$, respectively. $m_{0}$ is the free electron mass. The value for $K^{2}$ applies for the fast transverse wave propagating along the [110] direction of GaAs. The drift parameter is $v_{d} / v_{s}-1=5$. On the same figure, we have plotted (curve 2) the gain factor as calculated from Eq. (20) with $a=0$. This curve corresponds to the predictions of a semiclassical microscopic theory for a semidegenerate electron gas. For angular acoustic frequencies above $5 \times 10^{11} \mathrm{sec}^{-1}$, the probability that an electron possesses the momentum required to emit acoustic phonons is quickly decreasing, and the quantum mechanically calculated gain factor is drastically reduced. Finally, curve 3 shows the acoustoelectric gain factor as calculated semiclassically $(a=0)$ when neglecting the partial degeneracy of the electron gas $\left(q_{0}=q_{D}\right)$. On account of the reduced screening efficiency of the free carriers when the Pauli exclusion principle is taken into account, the frequency of maximum acoustic gain as well as the gain maximum itself are smaller for the 


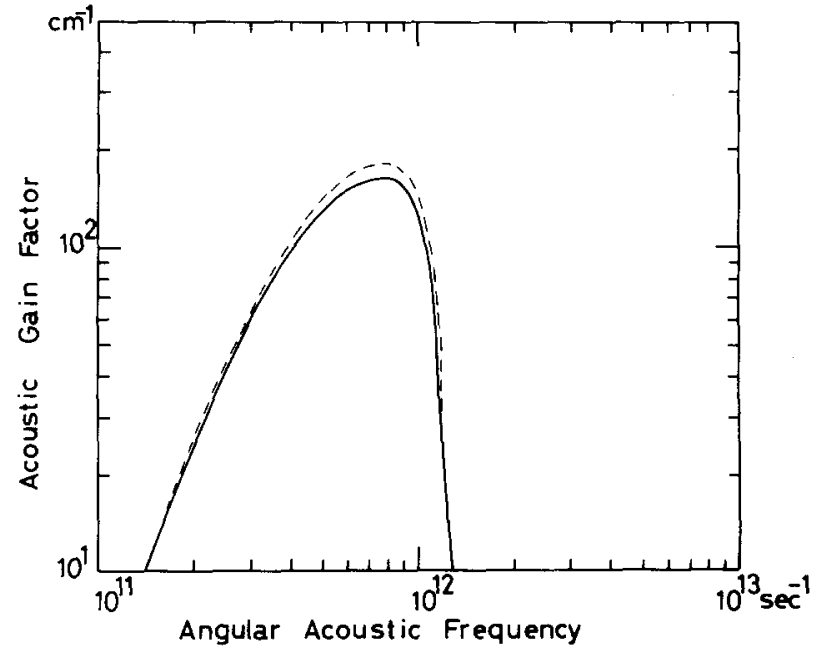

FIG. 2. Acoustic gain factor as a function of angular frequency for GaAs with a free-carrier density of $1.35 \times 10^{17} \mathrm{~cm}^{-3}$ at $20 \mathrm{~K}$. The electron gas is almost completely degenerate, and the rapid high-frequency cutoff of the gain factor reflects the sharpness of the Fermi surface. Dotted curve shows the results of the density matrix calculation by Gurevich and Kagan (Ref. 8).

semidegenerate electron gas than for a nondegenerate gas with the same density of carriers.

Figure 2 shows the acoustoelectric gain factor for a GaAs sample with a free-carrier density of $1.35 \times 10^{17}$ $\mathrm{cm}^{-3}$ at $20 \mathrm{~K}$. This sample is almost completely degenerate, and consequently the high-frequency cutoff is very sharp. As calculated from the simple theory outlined in this section, the gain factor agrees fairly well with the grain factor (dotted curve) obtained from a more elaborate density matrix calculation. ${ }^{8}$ The difference (12\% at the gain maximum) being a consequence of our neglect of the variation of the screening wave number with acoustic wave number. The high-frequency cutoff as calculated from Eq. (20) is not quite as sharp as predicted by the density matrix calculation of Ref. 2 . This is because Carlson and Segmüller have assumed a completely degenerate electron gas and thereby neglected the thermal broadening of the Fermi surface. In calculating the curves of Fig. 2, we have assumed the same values for $m^{*}, K^{2}$, and $v_{d} / v_{s}$ as for Fig. 1 .

\section{EFFECTS OF IMPURITY BANDING}

In Sec. II our calculation of the acoustoelectric gain factor was based upon the rigid-band model which assumes the intrinsic band structure of the material to remain unaffected by the presence of impurities. It is well known ${ }^{9}$ that the overlap of bound electron wave functions associated with shallow impurity states for heavily doped semiconductors leads to the formation of impurity bands, which may distort the principle bands of the material. For intermediate impurity concentrations, where the overlap of the bound electron wave functions associated with neighboring impurity sites is still small, charge transport in the impurity band takes place through a phonon-assisted hopping process. ${ }^{18}$ In this doping range, the impurity band is relatively narrow and situated just below the main band from which the impurity states are split off. With increasing im- purity concentration, the impurity band widens and merges with the main band. A transition to a metalliclike conduction then occurs with the Hall coefficient becoming almost independent of temperature.

A precise account of the effects of impurity banding upon the accoustoelectric interaction is difficult to give. The energy and momentum conservation conditions for the electron-phonon interaction will be affected by the presence of itinerant electrons in impurity-band states. The screening wave number depends on the density-of-states function and on the position of the Fermi level, both of which are affected by the presence of impurity-band states close to the conduction-band edge. Finally, the matrix element for electron-phonon coupling may change if the electronic wave functions in the presence of an impurity band deviate considerably from simple plane waves. In the doping regime where the conduction is metallic-like, electrons occupying impurity band states in many respects behave like free electrons with an effective mass and a mobility comparable to the mass and mobility of electrons in the intrinsic conduction band. ${ }^{19,20}$ This is the regime that we are going to discuss in the present section, taking at the outset the simplest possible model. For GaAs, the transition from phonon-assisted hopping to metallic-like conduction takes place at a donor concentration of about $4 \times 10^{16}$ $\mathrm{cm}^{-3} \cdot{ }^{21}$

We shall assume that the itinerant electrons of our highly doped semiconductor can be divided into two categories: conduction-band electrons and impurity-band electrons, each of which (for a restricted range of energies and momenta) can be described within the effective mass approximation. With the conduction-band electrons we associate an effective mass $m^{*}$, and with the impurity -band electrons an effective mass $m_{d} \equiv g m^{*}$. Taking the energy of a zero-momentum conductionband electron as origin of energy, the energy of an impurity-band electron of zero momentum is assumed to

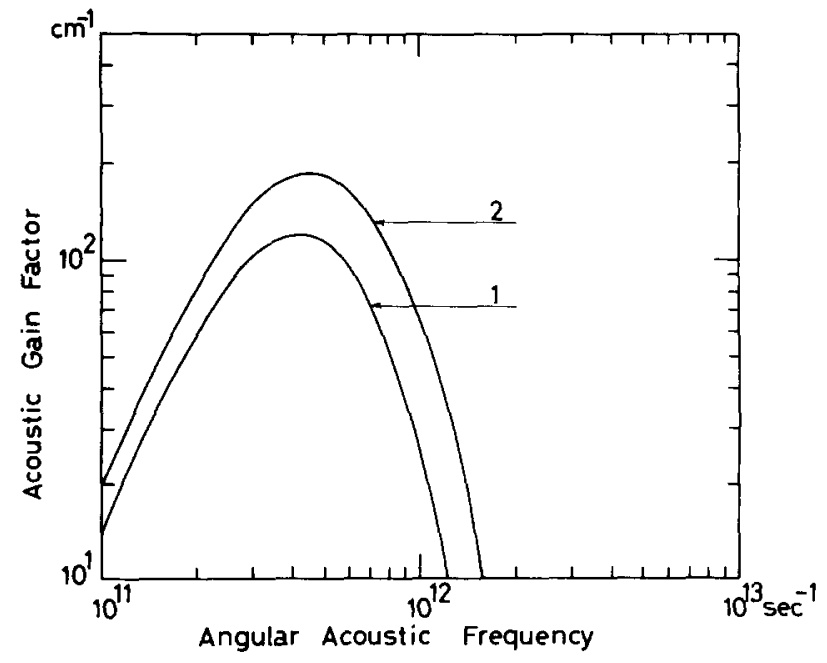

FIG. 3. Acoustic gain factor as a function of angular frequency for GaAs with a free-carrier density of $4 \times 10^{16} \mathrm{~cm}^{-3}$ at $77 \mathrm{~K}$. Curve 1 was calculated assuming all mobile electrons to be conduction-band electrons. Taking the presence of an impurity band into account (curve 2) leads to an enhancement of the acoustic gain and to a broadening of the active acoustic spectrum. 
be $\Delta \equiv-b k_{B} T$. It is further assumed that the electronic wave functions for both bands are plane-wavelike. For intraband electron transitions, the matrix elements for electron-phonon coupling will then be identical for the two bands and will be given by Eq. (4). Interband transitions are neglected. Finally, it is assumed that the mobility ratio for the two kinds of electrons is equal to the inverse mass ratio.

With this simple two-band model, the acoustoelectric gain factor can be recalculated following the procedure outlined in Sec. II. We obtain

$\beta=\sqrt{\pi} \frac{K^{2} \omega}{v_{T}} \frac{q^{2} q_{c}^{2}}{\left(q^{2}+q_{1}^{2}\right)^{2}}\left[f_{0}(a)\left(\frac{v_{d}}{v_{s}}-1\right)+g^{2} f_{0}\left(\frac{a}{g}-b\right)\left(\frac{v_{d}}{g v_{s}}-1\right)\right]$,

where the screening wave number $q_{1}$ is given by

$$
q_{1}^{2}=q_{c}^{2}\left[F_{-1 / 2}(\eta)+g^{3 / 2} F_{-1 / 2}(\eta+b)\right] .
$$

In the two-band model, the position of the Fermi level $E_{F}=\eta k_{B} T$ can be obtained from the relation.

$$
n_{0}=N_{c}\left[F_{1 / 2}(\eta)+g^{3 / 2} F_{1 / 2}(\eta+b)\right],
$$

where $n_{0}$ is the total density of free electrons. As before, $N_{c}=2\left(m^{*} k_{B} T / 2 \pi \hbar^{2}\right)^{3 / 2}$ is the effective density of states for the conduction band, and $a=\hbar^{2} q^{2} / 8 m * k_{B} T$ is the energy of a conduction-band electron having a wave number of $\frac{1}{2} q . v_{T}=\left(2 k_{B} T / m^{*}\right)^{1 / 2}$ is the thermal velocity, $v_{d}$ the drift velocity of the conduction-band electrons, and $q_{c}=\left(e^{2} N_{c} / \epsilon k_{B} T\right)^{1 / 2}$.

According to our assumption of a mobility ratio of $g$ for the two kinds of electrons, the average drift velocity is

$$
\begin{aligned}
v_{D}= & v_{d}\left[F_{1 / 2}(\eta)+g^{1 / 2} F_{1 / 2}(\eta+b)\right] \\
& \times\left[F_{1 / 2}(\eta)+g^{3 / 2} F_{1 / 2}(\eta+b)\right]^{-1} .
\end{aligned}
$$

It is noticed that a simple expression for the threshold drift velocity for acoustic amplification no longer exists.

In Fig. 3 we have plotted the acoustoelectric gain factor as a function of angular acoustic frequency for a GaAs sample having a free-carrier density of $n_{0}=4 \times 10^{16}$ $\mathrm{cm}^{-3}$ at $77 \mathrm{~K} .^{1}$ For the effective conduction-band mass and the squared electromechanical coupling constant, we have assumed $m^{*}=0.067 m_{0}$ and $K^{2}=3.7 \times 10^{-3}$, respectively. The drift parameter is $v_{D} / v_{s}-1=5$. Curve 1 shows the gain factor as calculated from $\mathrm{Eq}$. (20), neglecting effects of impurity banding. Curve 2 has been calculated from Eq. (25), assuming a mass ratio of $g$ $=2$ and a normalized band-edge separation of $b=0$. These values for $g$ and $b$ have been chosen fairly arbitrarily, the purpose of the present calculations being mainly to illustrate general trends in the effects of impurity banding upon the acoustoelectric interaction.

As can be seen from Fig. 3, taking impurity banding into account gives rise to an enhancement of the acoustoelectric gain and a broadening of the active acoustic spectrum. At the same time, the frequency of maximum gain is shifted slightly upwards. These effects of the impurity band upon the acoustoelectric interaction can be related to the increased density of states near the conduction-band edge and to the higher momentum effective mass of the impurity-band electrons. One would the refore not expect these effects to be consequences of our particular model. The presence of impurityband states close to the conduction-band edge leads to a reduction of the degree of degeneracy for the electron gas. With a less degenerate electron gas, the frequency of maximum gain as well as the gain maximum itself are increased, as discussed in relation with Fig. 1. The high-frequency cutoff of the gain factor occurs when the phonon momentum exceeds twice the typical electron momentum. With a somewhat higher momentum effective mass for impurity-band electrons than for conduction-band electrons, the impurity-band electrons will have a higher thermal momentum, and the high-frequency cutoff will be shifted upwards by their presence.

Some experimental evidence exists which supports our conclusions about the influence of impurity banding upon the acoustoelectric interaction. In highly doped GaAs samples, we have observed ${ }^{22}$ an enhancement of the acoustic gain relative to the predictions of the rigidband model in measurements of the incubation time for acoustoelectric current saturation. At $77 \mathrm{~K}$, the enhancement was typically about $30 \%$ for samples having a free-carrier density of $n_{0}=5 \times 10^{16} \mathrm{~cm}^{-3}$. Measurements ${ }^{1}$ of the spectral distribution of amplified acoustic flux in highly doped GaAs samples have shown a broadening of the spectrum relative to the predictions of the rigid-band model. Part of this broadening may be due to nonlinear acoustic amplification as suggested in Ref. 1. The theory of nonlinear acoustic interaction, ${ }^{23}$ however, predicts a fairly inefficient mode conversion at the high frequencies of the experiment $(50-100 \mathrm{GHz})$. It therefore seems reasonable to ascribe part of the broadening of the acoustic spectrum to effects of impurity banding.

Recently, the observation of a double peak in the spectrum of amplified acoustic flux has been reported for a GaAs sample having a free-carrier density of $n_{0}=1.35$ $\times 10^{17} \mathrm{~cm}^{-3}$ at $20 \mathrm{~K} .^{2}$ The primary peak, at an acoustic wave number of $q=1.85 \times 10^{6} \mathrm{~cm}^{-1}$, was found to occur at the frequency of maximum net gain predicted by the rigid-band model. The secondary peak at $q=2.7 \times 10^{6}$ $\mathrm{cm}^{-1}$ was attributed to parametric upconversion, and subsequent linear amplification, of low-frequency modes.

For certain values of the mass ratio $g$ and the reduced band separation $b$, the two-band model developed in the present section predicts a double maximum for the linear acoustic gain factor. Impurity banding therefore appears as an alternative explanation to mode conversion of the structure of the acoustic spectrum observed at these very high frequencies. The double maximum may arise at low temperatures and high doping levels because of a sharp cutoff of the acoustoelectric interaction with conduction-band electrons at relatively low frequencies, where the strength of the interaction with impurity-band electrons is still increasing with acoustic frequency. The cutoff frequency for the interaction with conduction-band electrons can be lower than the cutoff frequency for impurity-band electrons because of the band separation and the different effective masses which allow the Fermi momentum for the impurity band to be considerably larger than the Fermi momentum for the conduction band. 


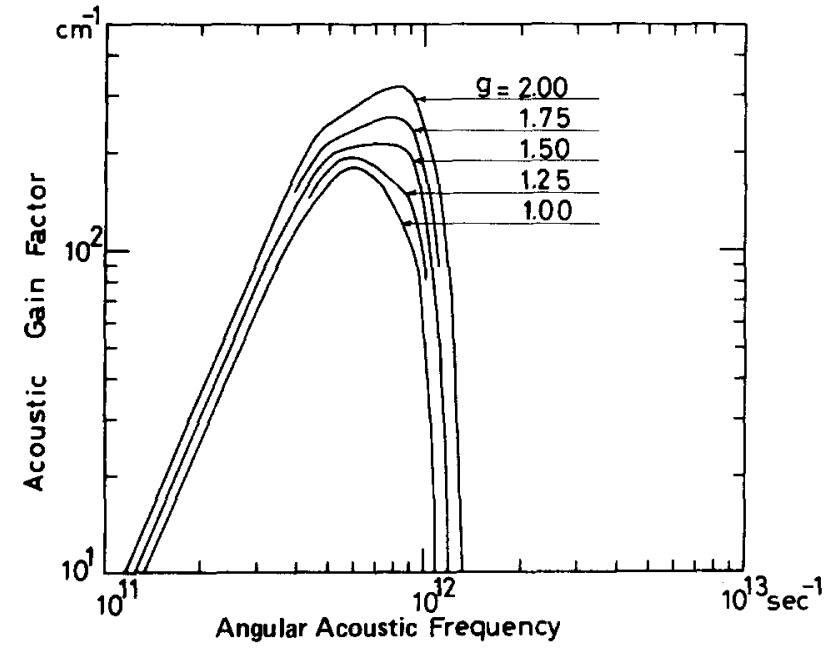

FIG. 4. Acoustic gain factor as a function of angular frequency for GaAs with a carrier density of $1.35 \times 10^{17} \mathrm{~cm}^{-3}$ at $20 \mathrm{~K}$. Effects of impurity banding are taken into account in a simple two-band model. $g$ gives the effective mass of an impurity-band electron in units of the conduction-band mass. The subsequent cutoff of the acoustoelectric interaction with conduction-band electrons at $6 \times 10^{11} \mathrm{sec}^{-1}$ and with impurity-band electrons at $9 \times 10^{11} \mathrm{sec}^{-1}$ gives rise to the shoulders of the gain curves.

In Fig. 4 we have plotted the acoustoelectric gain factor as a function of angular acoustic frequency with the mass ratio $g=m_{d} / m^{*}$ as a parameter. The five curves were calculated from Eq. (25) with $n_{0}=1.35 \times 10^{17} \mathrm{~cm}^{-3}$ and $T=20 \mathrm{~K}$. The usual values for $m^{*}, K^{2}$, and $v_{D} / v_{s}$ were employed. For the reduced band separation, we have chosen the value $b=3.5$. This value for $b=-\Delta /$ $k_{\mathrm{B}} T$ corresponds to the ratio $E_{d} / k_{B} T$ of the donor ionization energy to thermal energy if for the donor ionization energy for GaAs one inserts the value $E_{d}=6 \mathrm{meV}$.

As can be seen from Fig. 4, for the assumed values of the various parameters, the cutoff of the interaction with the conduction-band electrons is not quite sharp enough to produce a double peak for the gain factor. For mass ratios between 1.25 and 1.75 , however, the cutoff is sufficiently rapid to produce a fairly sharp knee on the gain curve at an angular acoustic frequency of $\omega$ $=6 \times 10^{11} \mathrm{sec}^{-1}$. For $g=1.50$, the acoustic gain is virtually constant over the range of angular frequencies from 5.5 to $9.0 \times 10^{11} \mathrm{sec}^{-1}$, corresponding to the range $(1.65-2.70) \times 10^{6} \mathrm{~cm}^{-1}$ for the acoustic wave number. This wave number range covers with reasonable accuracy the range around the observed double maximum for the acoustic spectrum. ${ }^{2}$ To produce a double maximum for the theoretical gain curve, we would have to assume a somewhat lower temperature or a larger value for the band separation. On the other hand, the experimental accuracy as indicated in Fig. 1 of Ref. 2 is barely sufficient to distinguish between the presence of two maxi$\mathrm{ma}$ in the spectral distribution and the presence of just a flat top.

If the temperature is reduced to $10 \mathrm{~K}$, the double-peak structure clearly appears in the theoretical gain curve. This is shown in Fig. 5. The curves of this figure were calculated assuming the same values for $n_{0}, K^{2}, m^{*}$, and $v_{D} / v_{s}$ as in Fig. 4. For the reduced band separation we have taken $b=7.0$ corresponding to $E_{d}=6 \mathrm{meV}$ and
$k_{B} T=0.87 \mathrm{meV}$. Below $6 \times 10^{11} \mathrm{sec}^{-1}$, the acoustic wave interacts simultaneously with electrons of the two bands. At $6 \times 10^{12} \mathrm{sec}^{-1}$, the cutoff of the interaction with the conduction-band electrons sets in, and above $7.5 \times 10^{11}$ $\mathrm{sec}^{-1}$ only impurity-band electrons take part in the acoustoelectric interaction. Finally, at an angular acoustic frequency of about $10^{12} \mathrm{sec}^{-1}$, the interaction with impurity-band electrons is also cut off. For mass ratios around $g=1.4$, the strengths of the interactions with the two kinds of carriers are balanced such that two maxima are produced on the gain curve.

\section{DISCUSSION}

In the analysis of acoustoelectric effects in highly doped piezoelectric semiconductors, it is necessary to take the degeneracy of the electron gas as well as impurity banding into account. If the acoustic wave number approaches the linear extension of the electron distribution in reciprocal space, quantum effects and electron correlation effects must also be considered.

Based upon the one-electron approximation, we have developed a quantum theory of acoustoelectric coupling for arbitrary degeneracy of the electron gas. To simplify the analysis, we have neglected electron collisions, and we have assumed the screening wave number to remain independent of the acoustic wave number.

Electron collisions will give rise to a broadening ${ }^{2}$ of the high-frequency cutoff of the acoustoelectric gain factor when the acoustic wave number exceeds the characteristic linear extension of the electron distribution in reciprocal space. In most practical cases, however, we expect thermal broadening (associated with the deviation of the Fermi distribution from a mathematical step function) to dominate over collision broadening. Neglect of electron collisions is also consistent with our evaluation of the gain factor to first order in the parameter $\left|v_{d}-v_{s}\right| / v_{T}$, because only as long as colli-

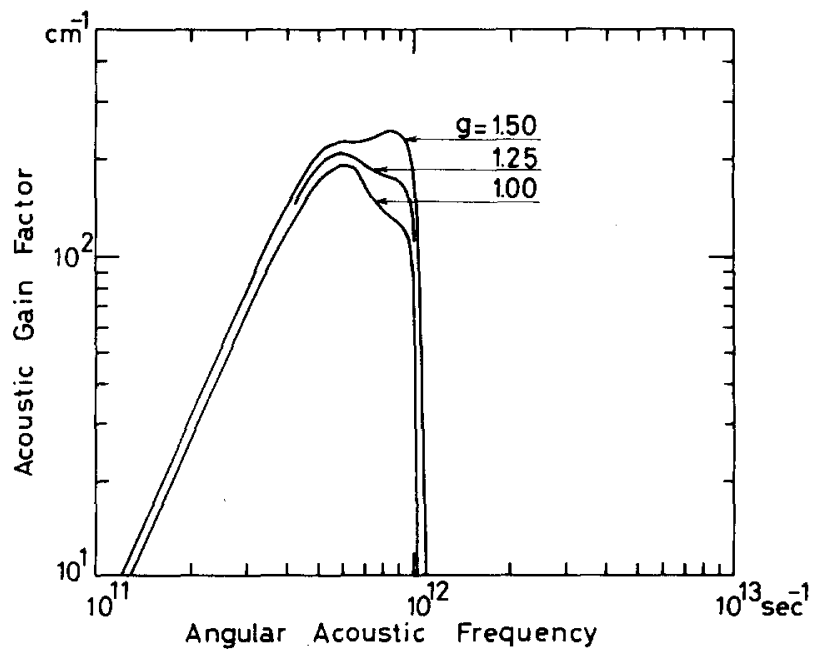

FIG. 5. Acoustic gain factor as a function of angular frequency for GaAs with a carrier density of $1.35 \times 10^{17} \mathrm{~cm}^{-3}$ at $10 \mathrm{~K} . g$ gives the effective mass of an impurity-band electron in units of the conduction-band mass. The rapid cutoff of the acoustoelectric interaction with conduction-band electrons at $6 \times 10^{11}$ $\mathrm{sec}^{-1}$ gives rise to the pronounced structure of the curves. For mass ratios of about 1.4 , the linear acoustic gain exhibits a double maximum. 
sions are neglected will the form of the gain curve remain independent of the electron drift velocity. ${ }^{24} v_{d}$ and $v_{T}$ are here the drift velocity and the thermal velocity of the free electrons, respectively; $v_{s}$ is the velocity of sound.

By comparison with the density matrix calculation performed by Gurevich and Kagan, ${ }^{8}$ we have found that neglecting the variation of the screening wave number with acoustic wave number leads us to underestimate the gain factor at the frequency of maximum gain by typically $10 \%$. This is approximately the accuracy to which higher -order terms in $\left|v_{d}-v_{s}\right| / v_{T}$ can be neglected, and therefore the accuracy to which our theory holds. It is also the accuracy to which the expressions for the zero-field attenuation constant obtained by Gurevich and Kagan ${ }^{8}$ and by Carlson and Segmuller ${ }^{2}$ can be employed to calculate acoustic gain factors. Besides, in the wave number range where the acoustoelectric interaction is strong (acoustic wave number smaller than the linear extension of the electron distribution in reciprocal space), we expect electron correlation ${ }^{25}$ to give rise to a weaker variation of the screening wave number with acoustic wave number than predicted in Refs. 2 and 8 on the basis of the one-electron approximation.

A simple criterion for the applicability of the one-electron approximation can be obtained by requiring that the average energy of the Coulomb interaction between two electrons be small compared to characteristic electron kinetic energies. ${ }^{8}$ Almost equivalent is the condition that the number of electrons within a screening sphere be large, i.e., $n_{0} / q_{0}^{3} \gg 1 . n_{0}$ is here the free-electron density and $q_{0}$ the screening wave number. Evaluating $n_{0} / q_{0}^{3}$ for a GaAs sample with $n_{0}=5 \times 10^{16} \mathrm{~cm}^{-3}$ at $77 \mathrm{~K}$ gives 0.05 . Hence, the one-electron approximation does not apply, and electron correlations should be considered. To the best of the author's knowledge, however, no attempt has so far been made to calculate the acoustic gain factor beyond the one-electron approximation. ${ }^{26}$

For many highly doped semiconductors, the most important correction to the simple one-band model of acoustoelectric amplification developed in Sec. II of the present paper is expected to arise from impurity banding. We have discussed a two-band model which treats conduction-band electrons as well as impurity-band electrons in the effective mass approximation. In spite of its extreme simplicity, this model is believed to reflect the major aspects of the effects of impurity banding upon the acoustoelectric interaction. These effects are the enhancement of the acoustic gain and the broadening of the active acoustic spectrum due to the increased density of states near the conduction-band edge and due to the presence of electrons of higher momentum effective mass than the conduction-band electrons. The two-band model seems to offer an alternative explanation for a number of experimental findings which have previously been ascribed to nonlinear interactions.

It is fairly simple to extend our model in a phenomenological way to permit the density-of-states effective mass for the impurity band to differ from the momentum effective mass, and to permit the matrix elements for electron-phonon interaction for the two bands to be different. In the present treatment, we have desisted from such a generalization, in order to avoid too many unknown parameters.

\section{ACKNOWLEDGMENTS}

It is a pleasure to acknowledge Dr. D. G. Carlson, Dr. J.C. McGroddy, and Professor P.N. Butcher for many helpful discussions. Professor N.I. Meyer is acknowledged for his interest in the work and for critical reading of the manuscript.

${ }^{1}$ D. G. Carlson, A. Segmüller, E. Mosekilde, H. Cole, and J. A. Armstrong, Appl. Phys. Lett. 18, 330 (1971).

${ }^{2}$ D. G. Carlson and A. Segmüller, Phys. Rev. Lett. 27, 195 (1971).

${ }^{3}$ W. Kohn, Phys. Rev. Lett. 2, 393 (1959).

${ }^{4}$ E. J. Woll, Jr. and W. Kohn, Phys. Rev. 126, 1693 (1962).

${ }^{5}$ K. Nakamura, Prog. Theor. Phys. 30, 919 (1963).

${ }^{6}$ B. V. Paranjape, Phys. Lett. 5, 32 (1963).

${ }^{7}$ E. Conwell, Phys. Lett. 13, 285 (1964).

${ }^{8}$ V. L. Gurevich and V. D. Kagan, Sov. Phys.-Solid State 4, 1788 (1963).

${ }^{9}$ N. F. Mott and W. D. Twose, Adv. Phys. 10, 107 (1961).

${ }^{10}$ A. B. Pippard, Philos. Mag. 8, 161 (1963).

${ }^{11}$ For momentum to be conserved in individual electron-phonon interactions, we must have $q l>1$ so that an electron can travel several acoustic wavelengths between two collisions. $l$ is here the electron mean free path. The assumption of momentum conservation therefore restricts our calculations to the regime $q l>1$. Formally imposing the condition of energy conservation upon the individual electron-phonon interactions, in principle, similarly restricts the theory to $\omega \tau \gg 1 . \tau$ is the electron collision time. In practice, however, the condition $\omega r>1$ can be relaxed so that our results remain good approximations independent of $\omega \tau$.

${ }^{12}$ See J. H. McFee, Physical Acoustics (Academic, New York, 1966), Vol. IV, Part A, pp. $1-45$, and references therein.

${ }^{13}$ See, for example, J. M. Ziman, Electrons and Phonons (Oxford U.P., London, England, 1960), chapter V.

${ }^{14}$ C. Jacoboni and E. W. Prohofsky, Phys. Rev. B 1, 697 (1970).

${ }^{15}$ See, for example, J. Blakemore, Semiconductor Statistics

(Pergamon, New York, 1962), Appendix C.

${ }^{16}$ R. B. Dingle, Philos. Mag. 46, 831 (1955).

${ }^{17}$ H. N. Spector, Phys. Rev. 165, 562 (1968).

${ }^{18}$ A. Miller and E. Abrahams, Phys. Rev. 120, 745 (1960).

${ }^{19}$ Assuming the impurity-band atoms to form a simple cubic lattice with lattice constant $a_{d}$, the effective mass of the impurity-band electrons can be estimated from the expression $m_{d}=6 K^{2} / a_{d}^{2} W$ which determines the effective mass of tightly bound electrons in the bottom of a narrow band of width $W$. [See, e.g., A. H. Wilson, The Theory of Metals (Cambridge U.P., Cambridge, England, 1954), Sec. 2.6.] Assuming the bandwidth to equal the donor ionization energy for $\mathrm{GaAs}$, i.e., $W=6 \mathrm{meV}$, when, for a donor density of $N_{d}=4 \times 10^{16} \mathrm{~cm}^{-3}$, the transition to metallic-like conduction occurs, we calculate $m_{d} \simeq 0.09 m_{0}$, or $m_{d} \simeq 1.3 m^{*}$. ${ }^{20}$ O. V. Emel'yanenko, T. S. Lagunova, D. N. Nasledov, and G. N. Talalakin, Sov. Phys.-Solid State 7, 1063 (1965).

${ }^{21}$ J. Basinski and R. Olivier, Can. J. Phys. 45, 119 (1967).

${ }^{22}$ E. Mosekilde, D. G. Carlson, and A. E. Blakeslee (unpublished).

${ }^{23}$ N. Mikoshiba, J. Phys. Soc. Jap. 20, 2160 (1965).

${ }^{24} \mathrm{E}$. Mosekilde (unpublished). A similar statement holds for the semiclassical microscopic theory: see, e. g., Ref. 17.

${ }^{25}$ L. J. Sham, Proc. R. Soc. Lond. 283, 33 (1965).

${ }^{26}$ L. J. Sham (Ref. 25) considered electron correlation effects in his calculation of phonon dispersion curves for sodium. 